\title{
Mise à jour de la fiche signalétique de l'Écosse
}

Updating Scotland's ID documents

Jean Berton

\section{(2) OpenEdition}

1 Journals

Édition électronique

URL : http://journals.openedition.org/rfcb/4969

DOI : $10.4000 / \mathrm{rfcb} .4969$

ISSN : 2429-4373

Éditeur

CRECIB - Centre de recherche et d'études en civilisation britannique

Référence électronique

Jean Berton, « Mise à jour de la fiche signalétique de l'Écosse », Revue Française de Civilisation

Britannique [En ligne], XXIV-4 | 2019, mis en ligne le 25 novembre 2019, consulté le 27 novembre 2019

URL : http://journals.openedition.org/rfcb/4969 ; DOI : 10.4000/rfcb.4969

Ce document a été généré automatiquement le 27 novembre 2019.

\section{(c) $(1)$}

Revue française de civilisation britannique est mis à disposition selon les termes de la licence Creative Commons Attribution - Pas d'Utilisation Commerciale - Pas de Modification 4.0 International. 


\title{
Mise à jour de la fiche signalétique de l'Écosse
}

\author{
Updating Scotland's ID documents
}

Jean Berton

\section{Introduction}

1 Cette étude ambitionne de rendre hommage aux travaux que Jacques Leruez a menés sur l'Écosse dès après que celle-ci a traversé l'épreuve du premier référendum d'autodétermination en 1979 alors que la transformation de l'empire en Commonwealth touchait à sa fin. Dans L'Écosse, une nation sans État, il s'agissait d'en comprendre les tenants et les aboutissants. En 2000, il publiait une mise à jour dans L'Écosse, vieille nation, jeune État. Quelque vingt ans après cette parution, nous voulons apporter une modeste contribution à la compréhension de ce pays ami de la France.

2 Le temps d'une génération, les Écossais se sont interrogés sur l'identité de leur nation et sur la valeur de l'union avec l'Angleterre: le résultat a été la réouverture du Parlement d'Écosse le 1 juillet 1999. En 2019, la crise majeure que traverse le RoyaumeUni depuis le 23 juin 2016 a ravivé une grave question : cette crise aboutira-t-elle au divorce de l'Écosse et de l'Angleterre? La séparation des deux nations qu'envisage le SNP, pour incertaine que soit l'issue de la crise du Brexit, ne peut être qu'un long processus où sera remis en cause un nombre incalculable d'évidences, telles que les langues nationales.

3 La campagne pour le référendum de 1979 a mis en avant les questions existentielles inévitables : qui sommes-nous, d'où venons-nous, où voulons-nous aller? Et Jacques Leruez dans L'Écosse, une nation sans État (p. 10), posait la question de manière plus globale: "Comment peut-on être écossais?» Depuis une quarantaine d'années beaucoup de questions ont trouvé leurs réponses et, le 18 septembre 2014, à la suite de riches débats, les Écossais ont participé massivement au référendum sur l'indépendance. Mais si le questionnement n'est pas de nature à donner des réponses définitives, la fiche signalétique de cette vieille nation doit être mise à jour, dans une 
double approche diachronique et interdisciplinaire, surtout à l'intention des pays proches, dont la France qui est encore trop prompte à confondre Écosse, GrandeBretagne et Royaume-Uni.

4 Cette mise à jour ponctuelle de la fiche signalétique de l'Écosse se concentre sur trois points : premièrement, pour répondre à la question « qui est l'Écosse », nous abordons la question de la reconnaissance par l'identité patronymique et l'identité visuelle; deuxièmement, puisqu'il est question de mettre fin à l'union, nous interrogeons l'étatcivil; et, troisièmement, nous devons aborder la question délicate des langues indigènes qui sont un enjeu majeur de différences internes au Royaume-Uni.

\section{L'identification de l'Écosse}

5 L'Écosse a deux noms officiels, Scotland (en écossais et anglais) et Alba (en gaélique), et aussi des surnoms latins : Scotia et Caledonia. La nation a des représentations visuelles : la licorne, le chardon, le sautoir, Caledonia, Dame Scotia et, pour quelque temps encore, Elizabeth Regina. Il s'agira de préciser Caledonia et Dame Scotia qui semblent être actuellement délaissées ${ }^{1}$.

\section{L'identité patronymique}

6 La nation écossaise a un double patronyme, soit en anglais et écossais "Scotland", l'étymologie latine «Scotti» et anglaise «land» signifiant littéralement 'terre des Écossais', soit en gaélique moderne "Alba », l'étymologie latine "albus/a » signifiant littéralement 'blanche' parce que vues à distance, les montagnes des Hautes-Terres étaient couvertes de neige une majeure partie de l'année... En même temps la plupart des villes portent un double nom, à l'instar d'Édimbourg : Edinburgh, en anglais et Dùn Eideann, en gaélique.

7 L'Écosse jouit aussi d'une double dénomination ancestrale latine: Scotia et Caledonia. Le nom de Scotia dérive du nom des immigrés qui à partir du deuxième siècle sont venus d'Irlande et se sont implantés dans les Hébrides et le long de la côte ouest. Identifiée par les Romains sous l'appellation de «Scotti » cette peuplade a fini par dominer et faire disparaitre les Pictes. Leur territoire a été appelé «Scotia » dans les écrits en latin.

Le nom de Caledonia a été donné par les Romains, au premier siècle, parce que le massif montagneux (cal) des Hautes-Terres était vue comme une place forte imprenable (dunum) pour les légions. Scotia et Caledonia, en tant que dénominations d'un même territoire, ont été personnifiées au $\mathrm{XVI}^{\mathrm{e}}$ siècle pour Scotia, et au $\mathrm{XIX}^{\mathrm{e}}$ siècle pour Calédonia ${ }^{2}$. Ces deux représentations emblématiques de l'Écosse ont pris corps dans des contextes politiques très différents.

Alors que Henry VIII formulait le projet de créer le royaume de 'Bretayne', l'image de Britannia commençait à prendre forme depuis que Polydore Vergil composa avant 1515, et fit imprimer en 1534, Anglica Historia ${ }^{3}$. L'élite d'Écosse connaissait le personnage de Boudicca; il restait à lui inventer une homologue, fût-ce dans le registre ironique. Robert Wedderburn, dans sa Complaynt of Scotlande (1549), créa un personnage féminin, laissant son lecteur libre de faire des rapprochements avec Marie de Guise : « my name is callit the affligit lady dame Scotia $»^{4}$. De fait, ce personnage né dans un songe représente la 
nation et non pas la reine douairière qui n'avait pas encore reçu officiellement le pouvoir de régence.

In my dullit dreyme ande sopit visione $\mathrm{i}$ thocht

That ther aperit to me ane lady of excellent extractione

Ande of anciant genolygie makkand ane

Melancolius cheir for the grite violens that sche hed

Sustenit \& indurit ${ }^{5}$

La tête et chevelure qualifient l'apparition: "Hyr / Haistylye in the maist extreme exterminatione hyr / Hayr of the cullour of fyne gold vas feltrit \& trachlit / Out of ordour hingand ouer hyr schuldirs ${ }^{6}$. Sa couronne d'or est chancelante et sur le point de tomber sur la terre froide parce que les héritiers putatifs du trône se jalousent et se neutralisent à l'avantage de l'ennemi. Son bouclier (F54V) arbore un lion rampant, symbole de la royauté, qui est blessé de toutes parts parce que les forces armées sont désorganisées. Son manteau est un miroir du pays, il expose la perte de la richesse, de l'instruction et de l'activité économique.

11 Cette mater dolorosa porte un manteau sur lequel l'auteur distingue clairement trois parties : dans la partie supérieure on voit des pierres précieuses gravées et des signes de noblesse; dans la partie médiane sont imprimés des lettres et des nombres, des bonnes actions et des miracles; dans la partie inférieure sont brodées des représentations de bétail et de produits agricoles et industriels. Dans sa description, le narrateur répète qu'il parvient à peine à lire. Le lecteur comprend que cette vision apocalyptique de la nation traduit un manque de présence politique de l'Écosse, et la conséquence est catastrophique puisqu'il n'y a pas le moindre espoir de salut : il s'agit d'un transfert de l'ambiance générale de la nation vers le personnage qui la représente.

La description sommaire de l'accoutrement de 'Dame Scotia' est inspirée des portraits peu flatteurs qu'avait fait François Deserps avant de les emporter avec lui en France.

La sauvage d'Escosse.

Si tu mets l'œil dessus ceste figure

A celle fin que certain tu en soys,

C'est la sauvage au pays Escossoys,

De peaux vestue encontre la froidure. ${ }^{7}$

13 Ainsi, l'élément commun entre Dame Scotia et «La sauvage d'Escosse » est le manteau. Celui de Dame Scotia est plus élaboré pour servir l'intention satirique de Wedderburn: le rapprochement entre le vêtement symbolique de Dame Scotia et celui de « La sauvage d'Écosse » montre que le pouvoir royal symbolisé par le manteau se dégrade à un point tel que la royauté écossaise sera bientôt réduite à néant. Si le conflit dit de la cour brutale n'a pas été remporté par le pouvoir anglais, les conséquences en seront l'anéantissement du pouvoir écossais.

\section{L'identité visuelle}

Portés par la vague de la Renaissance, les Tudors ont ressuscité le concept de Britannia en lui donnant l'esprit de résistance de Boudicca, représentée sur un char tout près du Parlement de Westminster. Sa réalité esthétique s'est concrétisée au moment de l'union des parlements d'Angleterre et d'Écosse en 1707. On l'a ensuite parée de vertus morales, politiques et mythologiques en la représentant comme la Neptune de l'Empire. Britannia est représentée en guerrière romanisée : elle porte une toge, un casque de général, un bouclier, une lance ou un trident ${ }^{8}$. Parodiant l'allégorie de la Grande- 
Bretagne, William Hole a créé le portrait de Caledonia (1898) pour manifester la supériorité de la science à la lutte armée, bien que Caledonia se tienne prête à prendre les armes. En effet, Caledonia est vêtue comme un général romain. Elle a posé son glaive et son bouclier. Elle est assise dans l'encadrement d'une fenêtre. De la main gauche elle tient un grand livre, de la main droite elle repousse le rideau pour montrer un ciel étoilé. Force est de constater qu'aucun des portraits de Marie de Guise que l'on trouve à la Scottish National Portrait Gallery (SNPG) d'Édimbourg ne correspond ni à la Dame Scotia de Wedderburn, ni à la Caledonia de Hole. Cependant, Marie de Guise, Dame Scotia et Calédonia ont en commun les valeurs morales de courage et détermination et une grande lucidité sur l'état de la nation.

Avant de chercher à représenter une idée de la nation par la création d'un modèle, on a représenté l'État sous les traits du monarque. Le tableau de Jacques V et de Marie de Guise après leur mariage est censé être le portrait officiel du monarque et de sa seconde épouse. Cependant, il y a deux versions de ce double portrait: dans la première, appelée le tableau de Blair Castle, le roi et la reine sont assis derrière une table. À gauche, le roi est tourné vers son épouse ; et réciproquement la reine est tournée vers la gauche. La main droite du roi est posée sur un livre imprimé symbolisant l'instruction et le savoir et la main gauche du roi recouvre la main droite de Marie pour indiquer leurs statuts respectifs. Jacques $\mathrm{V}$ regarde son blason, le lion rampant, et celui des Guise. Dans la seconde version, réalisée entre 1554 et 1560 alors que Marie était Régente, le roi et la reine regardent vers la gauche, l'un et l'autre tiennent entre pouce et index une pierre précieuse portée en collier, attribut de pouvoir.

La SNPG d'Édimbourg conserve une vingtaine de portraits, réalisés après son décès, censés représenter Marie de Guise : ils sont accompagnés de commentaires, tels que " artist unknown » ou « a possibly different sitter ». Le portrait réalisé par Corneille de Lyon, qui a fait la réputation de Marie de Guise à travers les siècles, a été peint un siècle après la mort de la reine. Cependant, il faut garder en mémoire que, du temps de Marie de Guise, l'iconoclasme était encouragé par les presbytériens. Duncan Macmillan rappelle que Jacques V, en 1541, fit voter une loi contre cette pratique ${ }^{9}$ : "George Wishart preached in Ayrshire in 1545 against images and caused great damage. $»^{10}$

Si Jacques $\mathrm{V}$ a hérité de son père le goût des arts et le sens de leur importance, Duncan Macmillan reconnaît que la reine venue de France partageait cette vue : elle avait fait venir de France Pierre Quesnel qui fit un portrait ${ }^{11}$ accompagné de ce commentaire : " Mary of Guise (Lorraine), Queen of James V. 1515-60. Composed 'aetatis suae 35 / anno Domini 45 '. Wearing two brooches, one with the ' $M$ ' and the other ' $L$ ' ". La cour et les visiteurs étaient sous le charme de la reine régente qui, devenue veuve, ne céda pas aux avances anglaises, ni pour elle ni pour sa fille, dans le but d'une union des couronnes anglaise et écossaise, et peut-être lorraine... Melanie Clegg, dans Scourge of Henry VIII, the Life of Marie de Guise, met en avant cette obsession chez le roi anglais d'épouser sinon Marie de Guise du moins de marier son fils à la reine écossaise.

Les années passant, Marie de Guise a cédé la prééminence à sa fille, Mary Stuart, alias Mary I $\mathrm{I}^{\mathrm{e}}$ Stuart, alias Mary, Queen of Scots ${ }^{12}$, qu'il faut distinguer de Marie, reine de France parce que épouse de François II. Quant à Marie la Régente, on constate que tous les portraits de la reine, de son mariage à son décès, y compris le profil imprimé sur les pièces de monnaie ${ }^{13}$, la montrent séduisante, mais aucun ne peut être daté du fait de l'absence d'arrière-plan. 
19 L'héraldique développée lors du Moyen-Âge a mis en scène des animaux inspirés des fables héritées des auteurs latins et grecs. Et, en 1707, le mariage du triple lion passant ${ }^{14}$ anglais avec le lion et la licorne rampants écossais constitue une fable propre à générer des métaphores. Les personnages emblématiques d'une part et les animaux mythiques d'autre part illustrent dans leurs démêlées au fil du temps la complexité, parfois la férocité, des rapports entre les dirigeants. L'épopée du lion britannique se terminant théoriquement avec la fin de l'empire, il reste la geste du lion et de la licorne : deux animaux inconciliables puisque le premier relève de la réalité et le second du fantastique.

\section{L'Écosse entre mariage contesté, allégeance refusée et cohabitation insoutenable}

20 Le quiproquo est un art à cultiver : tandis que l'Angleterre adoptait le rôle du soupirant éconduit mais certain de son droit et de la sincérité de son projet d'union, l'Écosse ne cessait d'affirmer son droit à la liberté d'autodétermination depuis que Constantin II avait fait, le premier, acte d'allégeance (c. 927) au roi anglais Athelstan. Pour l'Écossais, ce n'était qu'une formalité sans réel fondement ; pour l'Anglais, c'était un engagement. Les successeurs d'Athelstan n'eurent de cesse de s'appuyer sur ce précédent pour parvenir à leur fin, à savoir fondre toutes les parties des îles britanniques dans Britannia. Mais à compter de 1295, les rêves d'expansionnisme sur le continent ont été contrariés par Philippe le Bel et sa Vieille Alliance. La suite a montré que l'entente franco-écossaise, malgré les déceptions, n'a eu de cesse d'entraver les initiatives d'union exclusive anglo-écossaise.

\section{Le contrat de mariage}

21 Il était inévitable que la mort de Jacques $\mathrm{V}$ quelques jours après la naissance de Mary, sa fille légitime, causât des remous dans le monde politique de l'Écosse.

Two factions began to form amongst the Scottish nobles [...] By and large, there was a faction that was pro-English and, in the case of some of the nobles, there was a desire to bring in a measure of Protestant Reformation, which James had firmly rejected. The second group preferred the traditional Auld Alliance with France, and were generally committed to maintaining the Catholic faith. However, affiliations were not black and white, and there were Protestants who looked towards France, and Catholics who believed an accord with England was appropriate. ${ }^{15}$

Alors que les traités de Greenwich conclus peu de temps après la mort de Jacques $V$, en décembre 1542, étaient dénoncés par les Écossais, Henry VIII estimait qu'il était en droit de lancer une campagne punitive - comme Édouard I en 1296 - pour contraindre l'Écosse à respecter les traités qui l'engageaient à marier Mary Stuart à son fils et héritier. Si la mort d'Henry VIII, le 28 janvier 1547, mit fin à la première phase de la crise, en revanche le régent, Somerset, se révéla fidèle aux intérêts d'Édouard VI :

Believing it to be his 'godly purpose' to 'enjoin the Queen and Council to deliver the young Queen to be suitably nourished and brought up with her husband, as a Queen of England,' Somerset launched his military campaign against the Scots in August 1547. The ideological justification for his invasion and subsequent occupation of Scotland was deeply rooted in terms of Anglo-British imperialism. In his Epistle Exhortatorie to the Scots (1548), for example, Somerset reasoned that 'as you and 
wee be annexed and ioyned in one Islande, so no people so like in manner, forme, language, and all condicion [...] should bee, like two brethren of one Islande of great Britayn', and 'if it wer possible one kyngdome not to be divided in rulers'. ${ }^{16}$ successeurs au trône après Mary ${ }^{22}$. Mais cela fut contesté par le cardinal Beaton, archevêque de Saint-Andrews, qui se prévalait de sa nomination par le défunt monarque au Conseil de régence. Quoi qu'il en fût, la compétition entre Beaton et Hamilton, qui avait été nommé gouverneur par le Parlement le 13 mars 1543, prit fin avec le décès du cardinal en mai 1546. Beaton s'était opposé à la réforme en Écosse, et Hamilton était à la tête de la faction protestante pro-anglaise. Mais en mai 1554, le palais de Holyrood fut pillé et en partie détruit par l'armée anglaise, ainsi que le souligne Annette Bächstädt ${ }^{23}$. Et Hamilton retourna son pourpoint en faveur de l'Écosse tandis que Marie de Guise s'activait pour la promotion des intérêts de la France. Elle veillait aussi aux intérêts dynastiques de sa fille : cela incluait la liberté de l'Écosse et la réactivation de la Vieille Alliance franco-écossaise. Ritchie précise, page 19: «The treaties contracting the marriage of Mary Stewart to Edward Tudor were revoked and largely on account of England's extremely harsh Scottish policy, that is to say the military invasions known as the 'rough wooings'». Les divisions internes étaient telles que les Écossais ne s'accordaient sur rien, pas même sur l'opposition à la présence militaire anglaise sur leur territoire. C'est ainsi que les troupes anglaises purent infliger des punitions aux Écossais pour leur manque de soutien au projet de création d'un grand royaume de 'Brytain'.

uttes intestines entre pro-Anglais et anti-Anglais dans la crise de la demande en mariage faite par l'Angleterre exaspéraient le peuple écossais qui n'avait pas oublié la menace qui est au cœur de la Déclaration d'Arbroath de 1320 :

Yet if [our monarch] should give up what he has begun, and agree to make us or our kingdom subject to the King of England or the English, we should exert ourselves at once to drive him out as our enemy and a subverter of his own rights and ours, and make some other man who was well able to defend us our King; for, as long as but a hundred of us remain alive, never will we on any conditions be brought under English rule. 
Malgré sa ferme et constante opposition à une union entre sa fille, reine des Écossais, et Édouard VI d'Angleterre, les choix politiques de Marie de Guise restaient contestables puisqu'elle s'appuyait sur le soutien du roi de France.

\section{La conquête par les armes} d'avec l'Église de Rome qui ajoutait une valeur selon Ritchie : « a Protestant dimension to the existing geographical, linguistic and cultural arguments in favour of Anglo-Scottish union which culminated in the treaties of Greenwich ${ }^{24}$. Mais les Écossais, qui n'ignoraient rien de l'annexion récente $\mathrm{du}$ pays de Galles, n'eurent pas davantage confiance dans les promesses de Somerset que dans celles d'Henry VIII, à savoir qu'il respecterait l'indépendance de l'Écosse et qu'il souhaitait simplement libérer le pays du joug imposé par le Pape et qu'il n'avait que de bonnes intentions en sous-entendant le respect du contrat de mariage signé en 1543. Et Donaldson de préciser : « and the English manifestos drew attention to the material advantages which would accrue from the godly purpose $»^{25}$.

Si aucun accord de mariage n'était possible, restait la solution de la conquête. Les attaques anglaises culminèrent avec le désastre de Pinkie Cleugh le 10 septembre 1547. Somerset fit construire des places fortes dans des lieux stratégiques : les envahisseurs créaient une enclave sur le modèle du «pale» en Irlande. Cette enclave devait permettre aux armées d'occupation de mener des expéditions dévastatrices dans le Lothian et les Marches écossaises, telle que celle qui eut lieu en février 1548: "The English did not spend long in Scotland, but as well as causing the usual destruction they managed to capture Haddington, a wealthy and strategically placed burgh in East Lothian $»^{26}$.

Marie de Guise allait se révéler femme de tête : convaincue de la faiblesse de l'armée écossaise et de celle de ses chefs, elle prit les choses en main et se tourna d'abord vers le roi Henri II de France. Alors qu'il préparait la force d'intervention, James Hamilton prit langue avec les Anglais, évoquant la possibilité d'un changement radical de sa politique en leur faveur. Mais Henri II, qui le connaissait bien, avait exigé qu'il lui confie son fils aîné en otage ${ }^{27}$. De plus, Marie avait persuadé le roi de France de lui donner en mariage une riche héritière : Henri II lui offrit même un duché... Par ailleurs, la noblesse d'Écosse reçut d'importants cadeaux par la même occasion. La corruption devenait endémique, ce qui attisait le courroux de John Knox.

31 La flotte française arriva au port de Leith le 16 juin 1548, et après avoir accueilli la délégation menée par d'Essé, la reine fit le nécessaire pour que sa fille partît en France, le 7 août 1548, sur un bâtiment français. Puis elle exhorta tous les Écossais en âge de prendre les armes de se rendre dans les campements écossais. Elle montra alors ses talents de commandement :

she mounted her horse and rode along the High Street of Edinburgh, stopping at every house to root out any deserters lurking inside. As soon as she found them, she asked them in Scots. 'Is it thus that you set a good example? Before God, if I had not seen you with my own eyes and if anyone had told me that you were like to forget your honour in this way, I would not have believed them I should have thought such a thing incredible, having praised you so much all my life for what I believed and still do believe to be true, that no nation on earth would equal your courage!' [...] According to Beaugué, the soldiers were thoroughly ashamed and set off at once to rejoin the Scottish army. ${ }^{28}$ 
me l'aurait fait Boudicca ${ }^{29}$, Marie de Guise monta sur son cheval pour se rendre à Haddington afin de soutenir les soldats écossais qui assiégeaient la ville occupée par les Anglais :

On the evening of Monday 9 July she decided to venture as far as St Mary's church which lay on the edge of Haddington, beside the River Tyne. [...] Guns from both sides were still firing but [...] she rode up to the back of the church. At that moment there was a thunderous explosion as the cannon inside the town fired in her direction. ${ }^{30}$

Les chroniqueurs rapportent qu'elle fut blessée avec quinze autres soldats, et qu'elle perdit connaissance. À cause de l'aide du corps expéditionnaire français en quelques semaines l'armée anglaise abandonna toutes les villes occupées en Écosse. Et en juin 1549, une autre troupe, à la demande de Marie de Guise, reprit l'île de Inchkeith dans l'estuaire de la Forth.

reine en titre, Mary Stuart, fut promise au dauphin par le traité de Haddington du 7 juillet 1548, mais le conflit dit de la cour brutale ne prit fin que le 24 mars 1550 lorsque Français et Anglais signèrent le traité de Boulogne dans lequel la paix avec l'Écosse était

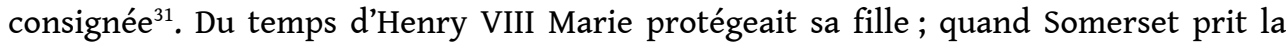
régence elle fut particulièrement active dans la défense de l'Écosse. Avant même qu'elle ne fût officiellement régente d'Écosse en 1554, Marie de Guise avait séduit les chroniqueurs qui montrèrent la reine veuve et mère sous les traits d'une vraie héroïne digne du titre de Dame Scotia. Selon Eric Durot ${ }^{32}$, Marie de Guise affirmait alors sa légitimité politique malgré le départ pour la France de sa fille déjà polyglotte.

En étudiant les événements qui ont touché l'Écosse au seizième siècle, il apparaît que la cohabitation entre l'Écosse, l'Angleterre et la France était cause de confrontation violente, durable conséquence de la guerre de Cent Ans. Marie de Guise puis Mary Stuart ont préservé l'indépendance de l'Écosse tant bien que mal. Suite à l'abdication de Mary Stuart et à sa soumission à Elizabeth Tudor en mai 1568, Jacques VI, fils de Mary et filleul d'Elizabeth, parvint à obtenir le trône d'Angleterre et contraignit l'Angleterre, l'Écosse et l'Irlande à une forme de cohabitation. Malgré l'union de 1707 et l'aventure conjointe de l'empire, la création du Royaume-Uni n'a pas apporté de solution pérenne puisque la Couronne se trouvait à la tête de trois nations - l'Angleterre (qui avait annexé le pays de Galles), l'Écosse et l'Irlande - peu enclines à la fusion.

En ce début du vingt-et-unième siècle, à la suite de la dévolution, même partielle et inégale, des pouvoirs, l'union britannique se transforme en cohabitation au sein de l'ensemble plus large de l'Union européenne, véritable mise en abyme. La crise du Brexit, qui n'est pas terminée à ce jour, met en évidence que le mariage entre l'Angleterre, l'Écosse et l'Irlande était un mariage à trois indésirable, dans la mesure où l'Écosse a voté à $62 \%$ pour rester dans l'Union européenne. On garde à l'esprit, comme une mise en abyme, que les Anglais, sinon les Britanniques, dans leur pratique quotidienne, n'ont jamais cessé de donner au mot 'Européens' le sens de 'Continentaux européens', s'excluant du concept d'union européenne.

\section{L'Écosse, une nation plurilingue}

Lorsque la religion chrétienne, avec le latin, s'est implantée en Écosse, à compter du sixième siècle, la carte linguistique était une mosaïque complexe. Des siècles plus tard, la langue des Anglo-Saxons n'a pas réussi à phagocyter les langues indigènes. Une 
certaine confusion vient de la cohabitation de langues nationales et de dialectes communautaires. Et devant la fermeté des Écossais quant à la reconnaissance et du gaélique et de l'écossais ( $\left(\operatorname{cots}^{33}\right)$, nous devons prendre pour acquis le fait que l'Écosse est une nation qui se revendique plurilingue, marque distinctive au sein de la GrandeBretagne.

\section{Un état des lieux}

Jacques Leruez, dans L'Écosse, une nation sans État (p. 43), place un intertitre provocateur, «Un non-problème : la langue », et ajoute : « Le traité d'Union ne mentionne pas la question de la langue. C'est qu'en 1707 la langue n'est déjà plus un problème». L'argumentation était recevable et discutable en 1979, mais une quarantaine d'années plus tard, le plurilinguisme n'est plus contestable. Leruez rappelle, à juste titre, p. 45 : « [...] comme ailleurs en Europe, un renouveau d'intérêt pour les langues minoritaires s'est manifesté en Écosse depuis les années cinquante." En effet, la Convention culturelle européenne de 1954 a été ratifiée par le Royaume-Uni en mai 1955. L'article 2 de cette Convention énonce :

Chaque Partie contractante, dans la mesure du possible :

[...]

2- s'efforcera de développer l'étude de sa langue ou de ses langues, de son histoire et de sa civilisation sur le territoire des autres Parties contractantes et d'offrir aux nationaux de ces dernières la possibilité de poursuivre semblables études sur son territoire. ${ }^{34}$

Cela signifie clairement que chaque langue a droit de cité dans son territoire et droit à la reconnaissance internationale. Il restera à définir ce qui est considéré comme langue et ce qui est considéré comme dialecte.

Il faut attendre 1992 pour que l'Europe publie à Strasbourg la Charte européenne des langues régionales ou minoritaires, ratifiée par le Royaume-Uni en 2001.

Article 1 - Définitions :

$\mathrm{Au}$ sens de la présente Charte: par l'expression «langues régionales ou minoritaires ", on entend les langues :

1- pratiquées traditionnellement sur un territoire d'un État par des ressortissants de cet État qui constituent un groupe numériquement inférieur au reste de la population de l'État; et différentes de la (des) langues(s) officielle(s) de cet État; elle n'inclut ni les dialectes de la (des) langues(s) officielle(s) de l'État ni les langues des migrants ; ${ }^{35}$

$\mathrm{Vu}$ la Charte européenne des langues régionales ou minoritaires, la question de l'utilité ou de la pertinence de l'écossais et du gaélique est caduque.

Britannia est un mot latin qualifiant les peuplades brittoniques d'avant l'invasion des Angles, des Jutes et des Saxons des cinquième et sixième siècle. Les Tudors ont repris le toponyme pour en faire un concept englobant l'emblématique Boudicca. Jacques VI d'Écosse et $\mathrm{I}^{\text {er }}$ d'Angleterre et d'Irlande a importé Scotia et Calédonia en 1603 et élargi le concept de Britannia à celui de Great Britain, zone anglophone. Mais contre toute attente, l'Écosse, partie prenante du Royaume-Uni, est restée une nation plurilingue : dans l'ordre chronologique, gaélophone, scottophone, anglophone.

43 À compter de la fin du haut Moyen-Âge, la nation écossaise s'est construite sur deux systèmes linguistiques inconciliables, celtique et germanique. Contrairement à la tendance générale, ni l'un ni l'autre n'a cédé la place et disparu; et l'Écosse clame que 
le plurilinguisme est un atout. Après avoir obtenu la majorité à Holyrood en 2007, le SNP a mis en avant la richesse linguistique de la nation ${ }^{36}$. Déjà, depuis avril 2005, le gaélique avait acquis la reconnaissance officielle en accédant au statut de langue nationale à parité avec l'anglais ; mais depuis la réouverture du Parlement, en 1999, les textes officiels sont publiés et en anglais et en gaélique ${ }^{37}$. Cependant, l'écossais, bien que parlé par une majorité d'Écossais, souffre à la fois de sa proximité d'origine avec l'anglais et de n'être pas encore parvenu à unifier la douzaine de dialectes présents sur le territoire ${ }^{38}$. Les scotticistes savent que l'insulte préférée des Écossais à l'endroit des Anglais est « Sassenach », qui ne signifie rien d'autre que Saxon...

\section{Le gaélique}

Donnée pour moribonde dès avant les années Thatcher, la langue gaélique s'est rénovée et unifiée grâce aux travaux de linguistes et à la volonté des politiques de préserver les langues mineures et régionales. Dans les années 1980, dictionnaires et manuels d'apprentissage ont été publiés pour établir les nouvelles normes grammaticales et orthographiques. Nombre de pédagogues et d'écrivains ont produit des textes adaptés à l'enseignement au niveau de l'école primaire, puis à celui du secondaire afin de créer un corpus à l'intention des élèves qui pourraient ainsi se former et passer des examens de valeur nationale. La production de nouvelles et de romans a été encouragée, ainsi que celle de pièces dramatiques. La poésie étant riche de sa variété depuis le quinzième siècle, il n'était pas indispensable de dynamiser l'inspiration des poètes. La reconnaissance de la langue, qui peut se mesurer au nombre d'enfants dans les écoles bilingues, a eu un effet salutaire sur la population gaélophone bilingue ${ }^{39}$. En effet, la confiance en soi et en sa langue maternelle a permis d'infléchir la tendance à quitter le territoire nationa ${ }^{40}$. Les cours d'été que dispense l'Université des Hautes Terres et des îles à l'intention des étudiants écossais et étrangers et les nombreux événements culturels redonnent à cette culture sa place dans le pays : le débat sur le nombre de locuteurs et le rôle que joue cette langue à part entière dans la nation n'est pas près de s'arrêter - il convient, cependant, d'estimer à leur juste valeur les faits, notamment donnés par les recensements, et l'effet, difficilement quantifiable. Quoi qu'on en pense, le gaélique écossais est une réalité aussi visible que le kilt et aussi audible que la cornemuse.

Les scotticistes français sont confrontés à des discours divergents animés de sentiments contradictoires : la négation pure et simple de la langue gaélique, son inutilité, la quasi impossibilité de l'apprendre, sa dépendance du folklore, mais aussi sa richesse littéraire, la différence de vision du monde... L'étudiant en études écossaises est avant tout placé devant une langue différente mais pas plus complexe que l'anglais. Une fois retenue l'évidence d'une nation trilingue, le latin mis à part, se pose la question légitime : où parle-t-on gaélique?

Le mot « Gàidhealtachd », simplifié en " Gaeltachd » (en écossais), traduit en anglais par «Gaeldom », correspond au mot "Gaélie », néologisme ${ }^{41}$ que j'ai introduit en 2003. Gaélie est la contraction de gaélophonie. Jusqu'au dix-neuvième siècle, le terme de géographie «Highlands » signifiant rien d'autre que Hautes Terres était donné pour équivalent de "Gàidhealtachd ", mot gaélique formé de «Gàidheal » et du suffixe " achd " servant à créer un concept. Les Hautes-Terres ${ }^{42}$ se sont dépeuplées au dixneuvième siècle, et la pratique du gaélique a grandement diminué à l'avantage du 
dialecte anglais Highland English. La plus grande densité de gaélophones se trouve sur la côte d'Argyll et dans les Hébrides ${ }^{43}$. Cependant, les universités de Aberdeen, Glasgow et Édimbourg ont un département de gaélique, la ville de Glasgow a reçu une forte migration de gaélophones et la ville d'Édimbourg abrite le Parlement d'Écosse où le gaélique a droit de cité au même titre que l'anglais. Il s'avère donc que l'on est susceptible de rencontrer un(e) gaélophone partout sur le territoire de l'Écosse, d'autant plus que la concurrence entre gaélophones et scottophones a cessé depuis plus d'une génération. De cette rapide observation, on déduit que les limites de la Gaélie sont théoriquement celles de l'Écosse. Et la notion de gaélophonie se justifie par le fait que cette langue s'étudie dans certains territoires de l'ancien empire britannique, tels que le Canada et la Nouvelle Zélande.

\section{L'écossais}

La langue écossaise (Scots) a survécu à un double handicap : le premier est une origine commune avec l'anglais, puisque le nord-anglien s'est ancré et développé dans le nordest $^{44}$ aux sixième et septième siècles, puis à l'écart des royaumes saxons et angliens à cause du royaume danois (Danelaw, circa 871-954); le second est une fragmentation dialectale cristallisée par les effets de la révolution davidienne à partir du douzième siècle. De la gémellité supposée de l'anglais et de l'écossais est née une compétition incessante où l'anglais cherche à prendre le dessus. De la fragmentation en une douzaine ${ }^{45}$ de dialectes répertoriés des Marches à l'archipel des Shetland, ces variations dialectales sont des marqueurs de singularité issue de mouvements migratoires historiques. D'un point de vue politique, cette fragmentation est un obstacle à la reconnaissance officielle du statut de langue nationale. Il reste que ce qui est une faiblesse pour les uns est une richesse pour les autres: le dorique, par exemple, témoigne d'une vitalité remarquable de la zone d'Aberdeen : ce dialecte n'hésite pas à s'approprier, par la traduction, des œuvres de langues étrangères, telles que des pièces de Molière (Le malade imaginaire sous la plume de J. Derrick McClure devient The Hypochondriak) et le Macbeth de William Shakespeare (qui, traduit par Lorimer \& Purves, devient The Bludie Tragedie o' Macbeeth).

Lorsque, au cours de la guerre de Cent Ans, la langue française a été bannie d'Angleterre à l'avantage de l'anglais (English) qui allait au seizième siècle subir les transformations que l'on connaît, l'Écosse a affirmé sa propre langue en changeant l'appellation courante de «Inglis » à «Scottis » - et les Makars, tels que R. Henryson et W. Dunbar, lui ont donné ce socle formel que l'on trouve dans The Complaynt of Scotlande. La langue écossaise a continué de s'enrichir, bien au-delà de la Renaissance, de vocables français et latins, mais aussi gaéliques en les adaptant à sa phonologie. Au seizième siècle, l'écossais a subi les assauts de l'anglais pour des raisons politiques, et la période de la cour brutale à l'égard de Marie Stuart révèle que les Anglais cherchaient à discréditer la langue des Écossais en la rabaissant au niveau de "mauvais anglais». Mais on observe aujourd'hui une moindre persistance chez les anglophones à chercher à réduire à néant et la langue écossaise et la langue gaélique avec des arguments politiques culpabilisants.

49 On doit à Robert Burns (1759-1796) la revitalisation de la langue écossaise et le retour à la confiance en soi chez les scottophones et à Walter Scott d'avoir su mettre en avant la richesse linguistique de l'Écosse, notamment dans Waverley ${ }^{46}$ et les romans dits écossais 
(Waverley novels). Scott est resté sourd aux demandes de ses lecteurs anglophones anglais et américains de supprimer tout ou partie des pages écrites en écossais: il voulait exprimer sa vision du monde complexe en jouant des langues indigènes de son pays. Julian D'Arcy, dans Subversive Scott ${ }^{47}$, a montré qu'il était nécessaire de réévaluer l'œuvre de Walter Scott dans une perspective écossaise plutôt qu'anglo-britannique : ses romans écossais ne cachent rien, mais il est nécessaire de les aborder avec une sensibilité plurilingue pour percevoir les nuances d'ironie romantique ${ }^{48}$ dans son usage des langues et dialectes indigènes.

\section{L'anglais, l'écossais, le gaélique et le français} loi d'Union de 1707 - par la force dans les établissements scolaires ; jusqu'au milieu du vingtième siècle, les écoliers gaélophones et scottophones en ont conservé des souvenirs cuisants: l'usage de la ceinture comme fouet restant imprimé dans les mémoires plus longtemps que dans les corps. Aujourd'hui, en Écosse, un anglophone se distingue à la fois par son accent et par sa langue qui diffèrent de l'anglais dit d'Écosse, ou SSE (Scottish Standard English) qui veut se qualifier de langue de référence. Inévitablement, cette langue anglaise s'est fragmentée en différents parlers, dont le plus connu est l'anglais de Haute Écosse...

eure que, actuellement, la présence de l'anglais, de l'écossais et du gaélique crée une forme de cohabitation linguistique : c'est ce que l'on trouve exposé dans la revue annuelle New Writing Scotland qui a fait paraître son trente-cinquième numéro annuel en 2018. À la discrétion du responsable d'édition du numéro, on y trouve des formes courtes de prose, de poésie et de texte dramatique dans les trois langues indigènes: écossais, gaélique, anglais.

texte en moyen-écossais de The Complaynt of Scotlande de 1549 de Robert Wedderburn, recèle divers aspects de la crise que traversait l'Écosse. Le narrateur montre qu'il a une claire compréhension des événements politiques qui se déroulaient entre 1547 et 1549. Et l'on peut avancer que ce texte fondateur annonce les crises linguistiques futures. Le choix de Wedderburn d'écrire en écossais, la langue officielle de la Cour, est une décision militante: «oure Scottis language» souligne le fait que le terme «Inglis » a été abandonné au profit des voisins d'Angleterre. C'était une réponse aux envahisseurs du royaume voisin qui prétendaient que l'Angleterre et l'Écosse partageaient une même langue :

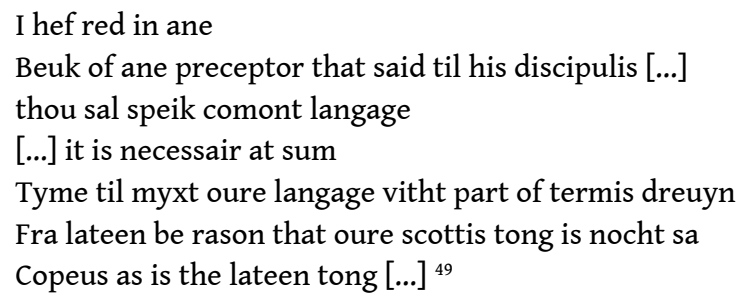

Alors que le latin était considéré comme la troisième langue d'Écosse, l'auteur mélange librement l'écossais et le latin; mais il n'autorise pas le personnage de Dame Scotia à s'exprimer en français, langue romane. L'emploi des langues sert le narrateur dans sa description de la souffrance des Écossais : «the langorius / desolat \& affligit pepil » (F1R). Ici, les trois termes qualifiant le peuple d'Écosse sont d'origine romane. Et la cause de cette dépression générale est la violence et la cruauté des Anglais qui sont traités de 
loups : « volfis of ingland» (F3R), autant pour exprimer un sentiment de mépris que pour donner au texte une saveur littéraire, puisque le loup était déjà le personnage cruel des fables et récits à valeur satirique.

L'intention de la complainte de Wedderburn est bien d'éveiller les consciences des lecteurs instruits par le biais des références abondantes aux civilisations anciennes: mais à quel tyran contemporain Wedderburn veut comparer Caligula?

[...] quha listis to reid the tragedeis of lucius seneque

or ihone bocchas in his buik of the ruyne of

nobillis thai sal fynd al cruel vsurpatours of vthir cuntreis

mak ane mischeuous ende there for $i$ hope in

god that vitht in schort days the protectour of ingland

and his cruel counsel sal be put in the croniklis in as

abhominabil stile as vas [...] Nero Callugala ${ }^{50}$

Les langues vivantes et anciennes sont la manifestation audible des enjeux politiques. Et la réforme religieuse oppose les langues vernaculaires aux langues classiques et amplifie le spectre de l'impérialisme linguistique apparenté à l'expansion romaine. Aujourd'hui, le traducteur de textes plurilingues se trouve devant une difficulté majeure car, ainsi qu'on le voit dans les romans écossais de Walter Scott et dans les textes de ses nombreux émules actuels, chacune de ces langues doit être traitée de manière distincte qui ne soit en aucun cas dépréciative. ${ }^{51}$

\section{Conclusion}

Aux touristes convaincus que l'Écosse est un pays celtique parce que longtemps considérée comme faisant partie de la «frange celtique », il convient de déployer une carte et de montrer la complexité onomastique - intégrant le nordique - du pays et sa richesse historique :

Which was the true Scottish nation? Was it the Gaeltachd, by then in the early nineteenth century confined to the Highlands and the Hebrides? Or was it the Scots- or English-speaking Lowlanders, who from the Middle Ages on had built up a rich culture of their own, a culture which in Walter Scott's heyday was influential in England and the English-speaking world, and also in Europe at large? ${ }^{52}$

Cette citation de John Pinkerton (1758 - 1826) interroge le concept de "Scottish nation » : «Scot » et «Scottish » ayant la même étymologie que quelque dix-huit siècles d'histoire ont rendue complexe en référant à la fois aux gaélophones, aux scottophones et aux anglophones, l'expression "Scottish nation » pourrait exprimer l'hybridité, tout en préservant une originalité. Il en résulte que la notion de communauté n'est pas étrangère aux Écossais qui la considèrent indispensable.

De George Buchanan (1506-1582) William Ferguson rappelle la subtilité de l'humanisme :

But although pro-French, his Epithalamium - celebrating the nuptials of the Dauphin Francis and the young Mary, Queen of Scots - also brags about the antiquity of the Scots and their fierce love of independence, hinting perhaps that, while the author preferred France to England, he yet had no wish for his country to fall under the French domination. ${ }^{53}$

La lucidité de George Buchanan sur les relations internationales de l'Écosse, que partageait Marie de Guise, est un héritage reçu par les responsables politiques d'aujourd'hui, comme le montre le comportement du gouvernement de Nicola Sturgeon 
sur l'émancipation de la nation. La paix dans l'archipel britannique leur importe grandement, ils observent, non sans inquiétude, l'agitation chez leurs voisins irlandais à propos de l'enclave anglo-britannique en Ulster. Oublieuse de Caledonia, Hibernia et Britannia, l'Écosse entend commercer avec l'Irlande comme avec les nations européennes, France incluse, comme avec l'Angleterre, mais ne pas être enfermée dans une relation dite privilégiée avec cette dernière. Est-ce à dire que les Écossais doivent s'attendre à une transformation de leur passeport britannique?

Jean Berton est professeur émérite en études écossaises de l'Université de Toulouse Jean-Jaurès. Son domaine de recherche interdisciplinaire couvre l'Écosse : auteur du Dictionnaire insolite de l'Écosse (2019), il aime dénicher des sujets méconnus et parcourir l'histoire de la nation en optant pour des angles de recherche propres à stimuler la curiosité de son lecteur. Il concentre actuellement ses études sur les langues nationales et la traduction de textes plurilingues.

\section{BIBLIOGRAPHIE}

Anonymous, Marie de Guise: Regent of Scotland (Coll. Tudor Times Insight, Tudor Times Ltd, 2015). $<$ www.tudortimes.co.uk> [14 octobre 2018].

Bächstädt, Annette, Maes, Bruno, Sukic, Christine, Marie de Lorraine-Guise, un itinéraire européen, Annales de l'est n 1-2017, Nancy, Université de Lorraine, 2018.

Brotchie, T.C.F., The Battlefields of Scotland, their Legend and Story, Édimbourg, T.C.\& E.C. Jack, 1913.

Clegg, Melanie, Scourge of Henry VIII, The Life of Marie de Guise, Barnsley, Pen and sword history, 2016.

D’Arcy, Julian, M., Subversive Scott, Reykjavik, Iceland University Press, 2005.

Deserps, François, Recueil de la diversité des habits, Paris, Bibliothèque Nationale de France, 1567.

Devine, Tom, et Logue, Paddy, Being Scottish, Édimbourg, Polygon, 2002.

Donaldson, Gordon, James V-James VII, Édimbourg, Oliver \& Boyd, (1965) 1978.

Ferguson, William, The Identity of the Scottish Nation, Édimbourg, Edinburgh University Press, 1998.

Fuga, Antonella, Artists' Techniques and Materials, Los Angeles, Getty Publications, 2006.

Keir, Dan, Machair, 2014, <https://www.poetrysoup.com/poem/machair_532726> [5 septembre 2019].

Leruez, Jacques, L'Écosse, une nation sans État, Lille, PUL, 1983.

Leruez, Jacques, L'Écosse, vieille nation jeune État, Crozon, Armeline, 2000.

Macmillan, Duncan, Scottish Art 1460-1990, Édimbourg, Mainstream, 1990.

Marshall, Rosalind K., Mary of Guise: Queen of Scots, Londres, Collins, 1977. 
Merriman, Marcus, The Rough Wooings Mary Queen of Scots 1542-1551, East Linton, Tuckwell, 2000.

Mikalachki, Jodi, The Legacy of Boadicea: Gender and Nation in Early Modern England, Abingdon, Routledge, 2014.

Moffat, Alistair, The Great Tapestry, Édimbourg, Birlinn, 2013.

Ritchie, Pamela E., Mary of Guise in Scotland, 1548-1560: A Political Study, Édimbourg, Tuckwell Press, 2002.

Schoentjes, Pierre, Poétique de l'ironie, Paris, coll. "Points/Essais-Inédits", 2001.

Trow, M. J., Boudicca: the Warrior Queen, Londres, Thistle Publishing, 2015.

Wedderburn, Robert, The Complaynt of Scotlande, 1549. Oxford Text Archive, <http://ota.ox.ac.uk/ desc/0388> [15 septembre, 2017].

Williams, H. Noel, The Brood of False Lorraine, the History of the Ducs de Guise 1496-1588, 2 volumes, Londres, Hutchinson \& Co, non daté.

\section{NOTES}

1. Cette étude ne traite pas des caricatures, telles que celle du troll, du 4 juin 1794, publiée sur la couverture de la revue de ESSE, Messenger, vol. VII, $n^{\circ} 2$, printemps 1998.

2. Depuis deux ou trois générations, Britannia est l'objet de raillerie sous la plume d'humoristes et de satiristes mais l'on ne cherche plus à recréer un portrait de Calédonia dont la physionomie soit reconnaissable.

3. Voir The Great Tapestry of Scotland, de MOFFAT Alistair (Édimbourg, Birlinn, 2013) : la page 74 représente le premier imprimeur d'Écosse, Chepman \& Myllar.

4. La traduction des extraits cités dans cet article ne sert qu'à guider la compréhension du texte. Les codes, ici F56V, indiquent les numéros de page. "On m'appelle Dame Scotia, la femme affligée ».

5. «Dans mon triste rêve et cette vision floue, j'ai cru / que m'apparaissait une dame d'excellente famille et d'ancienne lignée qui arborait / un air mélancolique suite à la grande violence qu'elle avait subie et endurée » (F54V).

6. «Sa coiffure dans un état lamentable / sa chevelure couleur d'or pur était crêpée / et tombait en désordre sur ses épaules » (F54V).

7. Images de DESERPS François. Texte et images de : <https://www.historyscotland.com/articles/ expert-history-articles/marie-de-guise-and-scottish-fashion-in-the-16th-century> Marie de Guise and Scottish fashion in the 16th century. Voir le site de BÄCHSTÄDT Annette: $<$ marieguiselorraine2015.com> [26 février 2019].

8. Ce concept androgyne a été secondé par le personnage mâle devenu légendaire de John Bull. Et John Arbuthnot, dans son ouvrage satirique The History of John Bull (1712), s'est bien gardé de faire de Peg, personnage féminin, son épouse: Peg est la sœur acariâtre de John. L'image de l'Angleterre et celle de l'Écosse s'en trouvaient à égalité d'origine, en théorie. Ainsi Britannia estelle le point de départ de la mythologie britannique, non pas anglo-saxonne parce qu'elle n'est pas germanique.

9. MACMILlan Duncan, Scottish Art 1460-1990, Édimbourg, Mainstream, 1990, p. 37.

10. Ibid., p. 38.

11. Coté à SNPG : S.Ph.I 38-20.

12. L'expression "Queen of Scots ", reine des Écossais, est la traduction littérale de l'expression latine utilisée pour la dynastie des Alpin (fondée vers 843) : Rex Scottorum. Dans la culture des Gaëls, le roi («Rì nan Albannach ») régnait sur des hommes et des femmes ; le peuple tout entier 
appartenait au territoire qu'ils occupaient et à qui ils allaient remettre leurs dépouilles, plutôt qu'ils possédaient la terre. C'est sous l'influence anglo-normande que les monarques devinrent " Kings of Scotland », "Rì na h-Alba », en gaélique moderne. La graphie en gaélique est variable au fil des générations : l'équivalent de « the Great Glen » est « Gleann Mòr », ou « Glen Albyn », la vallée de l'Écosse. À plusieurs reprises, Shakespeare place le personnage de Albany qui représente l'Écosse. Dans King Lear, les époux des trois filles représentent la France, l'Écosse et l'Angleterre.

13. Photo cotée à SNPG : S.Ph.I. 38-25.

14. Le léopard passant des Plantagenêts est devenu le lion passant au cours de la guerre de Cent Ans.

15. ANON. Marie de Guise: Regent of Scotland <www.tudortimes.co.uk> [14 octobre 2018], p. 15.

16. RITCHIE Pamela, Mary of Guise in Scotland, 1548-1560, Édimbourg, Tuckwell Press, 2002, p. 21.

17. Voir, dans The Rough Wooings, Mary Queen of Scots, 1542-1551, de MERRIMAN Marcus, l'étude détaillée de la crise. À la page 6, il résume l'événement: " The war took place in two stages : first under Henry VIII (from 9 November 1543 to 6 June 1546), then one directed by Edward's Protector, the Duke of Somerset (from 2 September 1547 until 24 March 1550). That actually makes two rough wooings. If one then includes the vigorous and successful war by the French King Henry II by which he achieved Mary's hand for his four-year-old son (June 1548 to April 1550), there are three wooings. "

18. MARSHALL Rosalind, Mary of Guise: Queen of Scots, Londres, Collins, 1977, p. 69.

19. RITCHIE Pamela, Mary of Guise in Scotland, 1548-1560, op. cit., p. 19.

20. WiLliams Noel, The Brood of False Lorraine, the History of the Ducs de Guise 1496-1588, Londres, Hutchinson \& Co, non daté, p. 99.

21. Voir l'article de BlAKeway Amy, "Marie de Guise: Dowager and Regent of Scotland » in BÄCHSTÄDT Annette (dir.), Marie de Lorraine-Guise, un itinéraire européen, Nancy, Université de Lorraine, 2018, p. 125.

22. Voir RITCHIE Pamela, Mary of Guise in Scotland, 1548-1560, op. cit., p. 17.

23. «Falkland Palace, Edinburgh and Holyroodhouse: Three Scottish Residences of Marie of Lorraine (1538-1559) » in BÄCHSTÄDT (dir.), op. cit., p. 117-129.

24. RITCHIE Pamela, Mary of Guise in Scotland, 1548-1560, op. cit., p. 19.

25. DONALDSON Gordon, James V-James VII, Édimbourg, Oliver \& Boyd, 1978, p. 77.

26. MARSHALl Rosalind, Mary of Guise: Queen of Scots, op. cit., p. 65.

27. Ibid., p. 66.

28. Ibid., p. 67.

29. Voir de MIKALACHKI J., The Legacy of Boadicea, Abingdon, Routledge, 2014.

30. MARSHALL Rosalind, Mary of Guise: Queen of Scots, op. cit., p. 68.

31. Ibid., p. 68.

32. « Marie de Guise, un sacrifice pour les siens », in BÄCHSTÄDT Annette, Marie de Lorraine-Guise, un itinéraire européen, op. cit., p. 69.

33. Afin d'éviter de m'exprimer en franglais inutilement, je préfère ne pas utiliser le terme "Scots» qu'il faudrait écrire «scotse " pour l'adapter à la grammaire et à la phonologie françaises. Le terme « écossais » signifiant « langue écossaise » n'offre pas d'ambiguïté.

34. <https://www.coe.int/fr/web/culture-and-heritage/european-cultural-convention>

35. <https://rm.coe.int/168007c07e>

36. Voir de Nihtinen Atina, « Gaelic and Scots in Devolved Scotland » (2008) sur le site : <https:// journal.fi/scf/article/view/7543> [1 ${ }^{\text {er }}$ juin 2019].

37. Voir de BERTON Jean, "Scottish Gaelic : An Age-Old Language in Modern Europe ", in LEYDIER Gilles (dir.) Scotland and Europe, Scotland in Europe, Cambridge, Cambridge Scholar Publishing, 2006, p. 142-155. 
38. Voir les travaux de ROBINSON Christine, lexicographe réputée, qui fut responsable de la mise à jour du Dictionary of the Scots Language. Elle est aussi l'auteure de Modern Scots Grammar (Édimbourg, Luath, 2012).

39. Il n'y a plus de locuteur gaélophone monoglotte depuis au moins deux générations. En revanche, s'il est difficile de quantifier au plus juste les gaélophones selon leurs compétences comme on le voit dans les recensements décennaux, on s'aperçoit qu'ils se sont libérés de toute la culpabilisation dont ils ont fait l'objet depuis des siècles. De surcroît, s'est imposée l'idée que le plurilinguisme est un atout. La gaélophonie concerne essentiellement : l'Écosse, le Canada et la Nouvelle-Zélande.

40. Voir, de Mccrone David, Understanding Scotland: The Sociology of a Stateless Nation, Londres, Routledge, 2001.

41. Voir «Gaélie ou Highlands, mythes et réalité ? », in Bernard SELLIN (dir.), Écosse des Highlands, mythes et réalités, Brest, $\mathrm{CRCB}$, Université de Bretagne occidentale, 2003.

42. Je distingue l'expression globalisante de Haute Écosse (sans trait d'union) de la partition géographique Haute-Écosse (Highlands), Basse-Écosse (Lowlands), Marches (Southern Uplands).

43. Le machair condense la vision du monde des gaélophones. Voir mon article «Le machair, symbole de la résistance victorieuse à la colonisation de l'Écosse septentrionale ", in BESSON F. (dir.) Écrits et cris de la terre dans le monde anglophone, Toulouse, PUM Caliban 61, octobre 2019, et le poème de Dan KEIR « Machair » : Where salt air, sand and soil meet; / A breeze of seasons sung a day. / Life grows to pass beneath your feet; / Still under grass we sleep to stay.

44. Les royaumes anglais de Bernicia (englobant le Lothian) et de Deira ont fusionné et se sont développés pour créer Northumbria (VIII ${ }^{\mathrm{e}}$ siècle).

45. Il va de soi que le nombre proposé par les linguistes est contesté.

46. Voir, de BERTON Jean, "Translating Scottish Literary Texts: A Linguistic Clover-Leaf » in CORBETT John (dir.), International Journal of Scottish Literature, Glasgow, IJLS, hiver 2010. Et « Le réalisme linguistique dans Waverley.» in sUнAмy H. (dir.), Scott Walter (Sir), Waverley, Paris, Ellipses, 1998.

47. D'ARCY, Julian, Subversive Scott, The Waverley Novels and Scottish Nationalism, Reykjavik, University of Iceland Press, 2005.

48. Voir, dans Poétique de l'ironie de P. Schoentjes, le procédé systématique de l'ironie romantique comme recours aux jeux énonciatifs qui brisent l'illusion romanesque par des commentaires décalés du narrateur ou des personnages. Ces commentaires souvent résident dans l'emploi d'un dialecte ou d'une langue différence de celle du narrateur. Selon F. Schlegel, l'ironie romantique, synonyme de liberté créatrice, permet à l'artiste de corriger le caractère subjectif de son œuvre par des moments objectifs. (<www.fabula.org/revue/cr/180.php>).

49. "J'ai lu dans un livre / d'un précepteur qui disait à ses élèves / [...] tu parleras la langue du peuple [...] il est nécessaire parfois / de mélanger dans notre langue des mots issus du latin / pour la bonne raison que notre langue écossaise n'est pas / aussi riche que ne l'est le latin [...] (F14V). 50. « [si tu] lis les tragédies de Lucius Sénèque ou Jean Boccace dans son ouvrage sur la ruine / de la noblesse tu trouveras tous les cruels usurpateurs des autres pays / qui ont connu une fin cruelle. Donc j'espère / - que le Seigneur m'entende - que très prochainement le roi d'Angleterre / et son cruel gouvernement seront présentés dans les chroniques / d'une manière aussi abominable que le sont [...] Néron et Caligula (F64V).

51. Voir «Étude sur The Spanish Galleon, de John Brandane (1921), drame archétypal écossais plurilingue », in BERTON J., CORRONS F., SURBÉZY A. (dir.) Traduction, plurilinguisme et langues en contact, Toulouse, PUM - « La main de Thôt » CETIM, juillet 2014.

52. FERGUSON William, The Identity of the Scottish Nation, Édimbourg, Edinburgh University Press, 1998, p. 309.

53. Ibid., p. 81. 


\section{RÉSUMÉS}

Le titre « Mise à jour de la fiche signalétique de l'Écosse » annonce une étude limitée à l'identité patronymique et visuelle de la nation, à l'origine et l'état de l'union avec l'Angleterre et au plurilinguisme assumé de l'Écosse. Les noms latins de Britannia, Scotia et Caledonia, hérités des Romains, forcent à explorer l'histoire sur quelque vingt siècles; la renaissance de Britannia a fait renaître Scotia au seizième siècle et la nouvelle tentative d'union a cristallisé leur antagonisme attisé par la France. Les langues indigènes de l'Écosse, enjeu de différence, loin d'avoir été diluées dans le Royaume-Uni se régénèrent depuis deux générations.

The theme of updating Scotland's documents developed in this article is centred on three points: the names and portraits of the nation, how valid the state of the union with England can be today (when compared to how powerful the idea of marriage was in the $16^{\text {th }}$ century), and how Scots and Gaelic have managed to survive the invasion of English. Since Britannia, Scotia and Caledonia are part of the Roman heritage, one must be prepared to be familiar with Scotland's history so as to evaluate the age-old antagonism between England and Scotland and the survival of the nation's indigenous languages.

\section{INDEX}

Keywords : Britannia, Caledonia, Scotia, union, multilingualism.

Mots-clés : Britannia, Caledonia, Scotia, union, plurilinguisme

\section{AUTEUR}

\section{JEAN BERTON}

Université Toulouse Jean-Jaurès, laboratoire CAS 